\title{
Analysis of Liver Tumors Using Preoperative and Intraoperative Contrast-Enhanced Ultrasound (CEUS/IOCEUS) by Radiologists in Comparison to Magnetic Resonance Imaging and Histopathology
}

\author{
Analyse von Lebertumorentitäten mittels präoperativer \\ und intraoperativer Kontrastmittelsonografie (CEUS) \\ IOCEUS) durch Radiologen im Vergleich zur \\ Magnetresonanztomografie und zur Histopathologie
}

\author{
Authors \\ Severin Huf ${ }^{1}$, Natascha Platz Batista da Silva ${ }^{1}$, \\ Isabel Wiesinger ${ }^{1}$, Matthias Hornung ${ }^{2}$, Marcus N. Scherer², \\ Sven Lang ${ }^{2}$, Christian Stroszczynski ${ }^{1}$, Thomas Fischer ${ }^{3}$, \\ Ernst Michael Jung ${ }^{1}$ \\ Affiliations \\ 1 Radiology, University Medical Center Regensburg, \\ Germany \\ 2 Surgery, University Medical Center Regensburg, Germany \\ 3 Radiology, University Medical Center Charité, Berlin, \\ Germany
}

Key words

CEUS, liver tumor, MRI, histopathology, IOCEUS

received 24.3.2016

accepted 29.11.2016

Bibliography

DOI http://dx.doi.org/10.1055/s-0042-124347

Published online: 2017 | Fortschr Röntgenstr 2017; 189:

431-440

(c) Georg Thieme Verlag KG Stuttgart · New York

ISSN 1438-9029

Correspondence

Prof. Ernst Michael Jung

Radiology, University Medical Center Regensburg

Franz-Josef-Strauß-Allee 11

93053 Regensburg

Germany

Tel.: ++ 49/941/9447410

Fax: ++ 49/9 41/9447409

ernst-michael.jung@ukr.de

\section{ABSTRACT}

Purpose To evaluate the diagnostic significance of preoperatively and intraoperatively performed contrast-enhanced ultrasound (CEUS/IOCEUS) in the diagnosis of liver tumors in comparison to magnetic resonance imaging (MRI) and histopathology.

Materials and Methods Retrospective analysis of 70/317 patients who underwent surgery for liver tumors between January 2012 and October 2015. Findings of CEUS and IOCEUS were compared to MRI. CEUS and IOCEUS were performed using multifrequency linear probes $(1-5,6-$ $15 \mathrm{MHz}$ ) after bolus injection of $1-5 \mathrm{ml}$ sulfur hexafluoride microbubbles. The histopathology after surgical resection, MRI morphology (T1, T2, VIBE, diffusion sequences) and wash-in/wash-out kinetics of CEUS were evaluated.

Results In 70 analyzed patient cases, 64 malignant liver lesions could be detected. 6 patients had benign liver lesions. Among the 64 malignant lesions, there were 28 metastases, 24 hepatocellular carcinomas (HCC), 9 cholangiocellular carcinomas (CCC) and 3 gallbladder carcinomas. 2 of the 6 benign liver lesions were hemangiomas, 2 were adenomas, 1 was an FNH and 1 was a complicated cyst. There was no significant difference when determining the lesion's malignancy/ benignity $(p=1.000)$. Furthermore, there was no statistical significance between preoperative CEUS and MRI regarding the general differential diagnosis of a tumor $(p=0.210)$ and the differential diagnosis classification between HCCs $(p=0.453)$ and metastases $(p=0.250)$. There was no statistical significance in tumor size $(10 \mathrm{~mm}-151 \mathrm{~mm}$; mean $49 \mathrm{~mm}$ SD +/$31 \mathrm{~mm}$ ) and location (tumor size $\mathrm{p}=0.579$; allocation to liver lobes $p=0.132$; segment diagnosis $p=0.121$ ) between preoperatively performed CEUS and MRI. The combination of preoperative MRI and CEUS for lesion detection showed significant differences compared to CEUS or MRI only ( $p<0.001$ for CEUS; $p=0.004$ for MRI). IOCEUS offered the substantial advantage of locating additional liver lesions ( $p=0.004$ compared to preoperative MRI, $\mathrm{p}=0.002$ compared to preoperative CEUS). In 10/37 cases (27\%) IOCEUS could locate further liver lesions which had not been identified during CEUS and/or MRI preoperatively, so that operative therapy was adapted accordingly and resection was extended if necessary.

Conclusion CEUS proves to be a dynamic imaging method for preoperative diagnosis of liver tumors showing high diagnostic significance in the characterization of a tumor's micro- 
vascularization, its entity and its size. During liver operations CEUS plays an important role in surgical therapy decisions.

\section{Citation Format}

- Huf S, Platz Batista da Silva N, Wiesinger I et al. Analyse von Lebertumorentitäten mittels präoperativer und intraoperativer Kontrastmittelsonografie (CEUS/IOCEUS) durch Radiologen im Vergleich zur Magnetresonanztomografie und zur Histopathologie. Fortschr Röntgenstr 2017; 189: 431-440

\section{ZUSAMMENFASSUNG}

Ziel Evaluation der diagnostischen Wertigkeit der präoperativen und intraoperativen Kontrastmittelsonografie (CEUS/IOCEUS) in der Lebertumordiagnostik im Vergleich zur Magnetresonanztomografie (MRT) und zur Histopathologie.

Material und Methoden Retrospektive Analyse von 70 Patienten, die aufgrund von Lebertumoren operiert wurden. Grundlage war die diagnostische Bildgebung mit CEUS und IOCEUS. Der Kontrastmittelultraschall wurde mit Multifrequenzsonden und Bolusinjektion von 1-5 ml SonoVue ${ }^{\circledR}$ durchgeführt. Anschließend wurden die Ergebnisse mit der MRT verglichen. Beurteilung der Histopathologie nach Resektion, der MRT-Morphologie und der Wash-in/Wash-out-Kinetik von CEUS.

Ergebnisse 64 von 70 Patienten hatten maligne Läsionen (28 Metastasen, 24 hepatozelluläre Karzinome (HCC), 9 cholangiozelluläre Karzinome (CCC), 3 Gallenblasenkarzinome). In 6 Fällen wurden benigne Läsionen histopathologisch gesi- chert (2 Hämangiome, 2 Adenome, eine FNH und eine komplizierte Zyste). Es konnten keine signifikanten Unterschiede in Bezug auf die Tumordignität $(p=1,000)$ zwischen präoperativem CEUS und der MRT festgestellt werden. Auch für die Differenzialdiagnose der Tumoren $(p=0,210)$ und die differenzialdiagnostische Einordnung von $\mathrm{HCC}(p=0,453)$ und Metastasen $(p=0,250)$ ergab sich keine statistische Signifikanz im Vergleich von präoperativem CEUS und der MRT. Für die Tumorgröße $(10 \mathrm{~mm}$ bis $151 \mathrm{~mm}$; im Mittel $49 \mathrm{~mm}$ SD $\pm 31 \mathrm{~mm}$ ) und die Tumorlokalisation ergaben sich ebenfalls keine signifikanten Unterschiede (Größenbestimmung $\mathrm{p}=0,579$; Lappenzuordnung $\mathrm{p}=0,132$; Segmentdiagnostik $p=0,121)$ zwischen präoperativem CEUS und der MRT. Die präoperative Kombinationsdiagnostik zur Herddetektion war im Vergleich zu jeweils einer der beiden Modalitäten signifikant überlegen ( $p<0,001$ für CEUS; $p=0,004$ für die MRT). Der IOCEUS bot den signifikanten Vorteil, zusätzliche Tumorherde zu erkennen ( $p=0,004$ im Vergleich zur MRT, $p=0,002$ im Vergleich zum präoperativen CEUS). In 10/37 Fällen $(27,0 \%)$ wurden durch IOCEUS weitere Herde histologisch gesichert.

Schlussfolgerung CEUS erweist sich in der präoperativen Diagnostik von Lebertumoren als eine dynamische Bildgebung mit hoher diagnostischer Aussagekraft über die Tumormikrovaskularisierung und ist somit hilfreich zur Bewertung von Tumorentität und Tumorgröße von Lebertumoren. Intraoperativ kommt CEUS eine wesentliche Bedeutung in der operativen Therapieentscheidung zu.

\section{Introduction}

To perform liver surgery successfully, even the smallest tumors must be reliably detected and characterized. Modern crosssectional imaging methods, particularly MRI with liver-specific contrast agent, have greatly improved the detection and characterization of liver tumors even in our own workgroups [1, 2], Numerous small lesions are visualized with MRI prior to surgery [2]. However, these lesions often require contrast-enhanced ultrasound (CEUS) for detection and characterization [3-11], but CEUS only achieves high diagnostic accuracy when performed by an experienced examiner [12 - 14].

Palpable tumors are additionally evaluated intraoperatively with ultrasound to decide whether resection of malignant lesions is necessary or whether a lesion appears benign [15].

In the case of deep lesions, it is often not possible to obtain findings via palpation. In the case of smaller non-cystic lesions, B-mode ultrasound alone is often not diagnostic [7, 16].

A DEGUM study showed the value of transabdominal liver tumor imaging with CEUS with a diagnostic accuracy for CEUS of $>90 \%[3-5]$.

Although intraoperative CEUS has been possible for over 10 years [17], it is only performed in individual cases by experienced examiners [15].

Inclusion of an experienced radiologist in a liver surgery team is valuable for helping to make decisions in critical cases regarding expanded resection due to tumor location and characterization based on intraoperative CEUS (IOCEUS) in combination with the preoperative diagnosis based on MRI using liver-specific contrast agent $[18,19]$.

The goal of the following studies is to determine the value of IOCEUS performed by an experienced radiologist compared to histology and preoperative imaging.

\section{Materials and Methods}

This retrospective study is based on 70 cases of patients who underwent partial liver resection between January 2012 and October 2015 at the University Hospital Regensburg. These 70 cases were selected from a total of 317 cases of patients who underwent partial liver resection in the indicated time period $(\triangleright$ Fig. 1).

247 cases were not included for the following reasons: Surgery was not indicated by a tumor in 24 cases. The histological finding was not definitive in 21 cases. 111 cases with no determination of clear malignancy were excluded based on the CEUS diagnosis. Instead of complementary imaging with CEUS and MRI, CT was used as the reference imaging method in 91 cases.

The inclusion criterion for the 70 selected cases was a tumor finding suspicious for malignancy as the indication for surgery with availability of preoperative CEUS and MRI findings for determining tumor status as complementary imaging. Moreover, a 


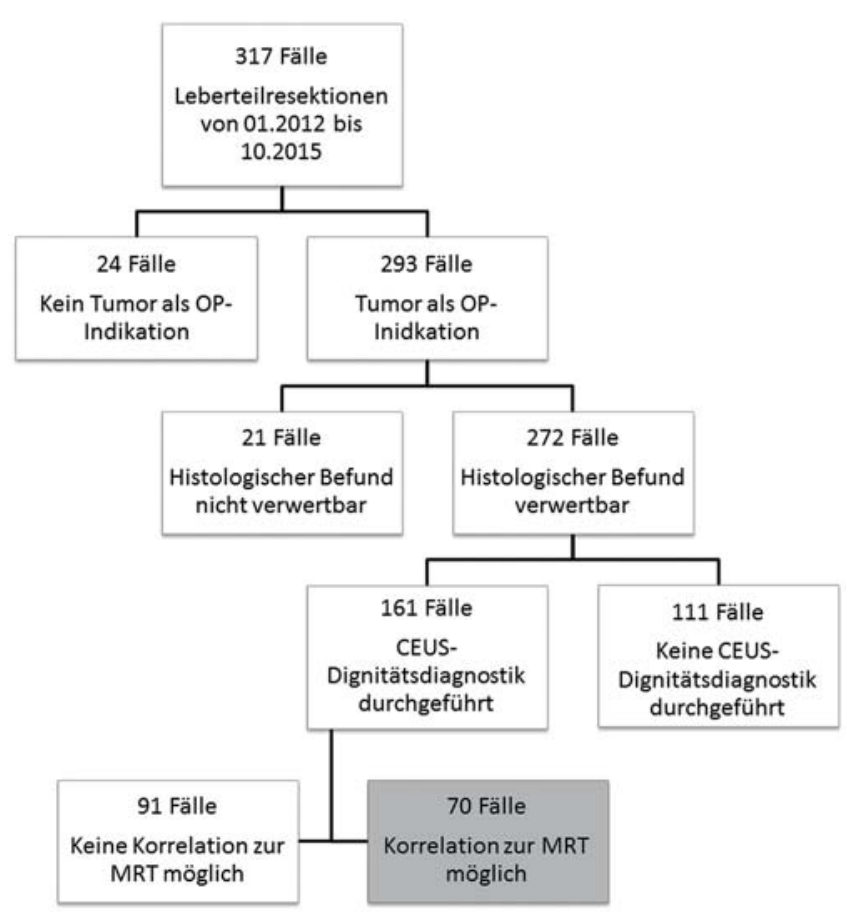

- Fig. 1 Decision flow chart for the 70 cases included in the study. 317 patients received (partial) liver resection between 01/2012 and $10 / 2015$ at the university medical center Regensburg. There was no tumor causing resection in 24 cases. In 21 cases the histopathological report was not useful. 111 cases were excluded because CEUS was not performed to determine malignancy/benignity. In 91 cases there was no additional imaging with CEUS/MRI. 70 cases were selected to determine the diagnostic value of CEUS and MRI in liver lesion.

histopathological finding had to be available as the gold standard for determining the status of liver tumors. Imaging was supplemented by IOCEUS in 42 cases.

Written informed consent was available for each examination. Every case was discussed regarding a surgical therapy decision in an interdisciplinary tumor conference. Approval of the local ethics commission (University Regensburg) was obtained for the study.

\section{Imaging techniques and examination procedure}

CEUS In preoperative CEUS, $1.0 \mathrm{ml}$ to $2.4 \mathrm{ml}$ contrast agent consisting of sulfur hexafluoride microbubbles (SonoVue ${ }^{\circledR}$, BRACCO) as a bolus with $10 \mathrm{ml} \mathrm{NaCl}$ were injected intravenously. In IOCEUS larger contrast agent quantities of $5-15 \mathrm{ml}$ SonoVue ${ }^{\circledR}$ were used. The higher intraoperative dose can be attributed to an increased destruction rate of the microbubbles in ventilated patients in connection with the oxygen content of the inhalation gas [20]. The correlation to other protocols also yielded a higher contrast dose under surgical conditions [19].

High-end ultrasound systems (LOGIQ E9/GE and Ascendus/Hitachi) were used. Preoperative CEUS examinations were performed with convex multifrequency transducers $(\mathrm{C} 1-5 \mathrm{MHz}$, C1 $-6 \mathrm{MHz}$ ), while multifrequency linear transducers ( $9 \mathrm{~L}$ transducer, 6-9 MHz; ML15 transducer, 6-15 MHz) with virtual adjustable convexity were used for IOCEUS. B-mode examination was followed by color-coded Doppler examination and power Doppler examination. CEUS was performed using a low MI technique (mechanical index $<0.16$ ) with amplitude modulation and phase inversion harmonic imaging (PIHI). Loops were documented in the arterial phase ( $15-45$ seconds), the portal venous phase (46 - 90 seconds), the venous phase ( $91-120$ seconds), and the late phase (3- 5 minutes). In particular, the constellation of irregular early arterial hypervascularization and wash-out beginning in the portal venous phase and increasing to the late phase was considered a main criterion for malignancy [15]. To differentiate HCCs from CCCs, the contrast agent dynamics were evaluated. In concordance with the current literature [21], HCCs show irregular hypervascularization in the arterial phase, contrast enhancement to the portal venous phase, and wash-out in the late phase. CCCs show contrast enhancement in the arterial phase at the margins and central hypoenhancement. Increasing wash-out with portal venous hypoenhancement to non-enhancement in the late phase is seen in CCCs.

MRI All MRI examinations were performed with a gadoxetate disodium solution (Primovist ${ }^{\circledR}$, BAYER) as the contrast agent with $5 \mathrm{ml}$ to $20 \mathrm{ml}$ being injected based on weight. A 3 T MRI system (Magnetom Skyra, Siemens) was used. Imaging was performed using the VIBE 3 D technique (Volume Interpolated Breathhold Examination) from the dynamic arterial phase (15-45 seconds) to the late phase (10-15 minutes). T1 and T2-weighted images with axial and coronal slices and a slice thickness of $5 \mathrm{~mm}$ were acquired. Adverse reactions to the contrast agent did not occur. However, one MRI examination had to be ended early due to claustrophobia so that only limited assessment was possible.

\section{Examiner and interpretation of images}

Preoperative contrast-enhanced ultrasound was performed by one experienced examiner (3000 ultrasound examinations per year for more than 10 years) at the interdisciplinary ultrasound center of the University Hospital Regensburg.

An experienced radiologist was responsible for all intraoperative ultrasound examinations. 1 of 5 surgeons specialized in liver surgery at the University Hospital Regensburg participated in each of the CEUS examinations.

An experienced MRI radiologist was responsible for interpreting the MRI examinations in consensus with various assistants.

\section{Statistical analysis}

The software SPSS (version 22.0, SPSS Inc., Chicago, USA) was used to create a database and for statistical evaluation. The significance analysis was performed with the McNemar and Wilcoxon tests and paired t-test. Values of $\mathrm{p}<0.05$ were considered significant.

\section{Results}

\section{Included cases and histopathology}

In the 70 included patients (43 men, 27 women, age 29 - 83 years, mean 62.9 SD +/- 11.9 years), the tissue for histopathological examination was obtained surgically.

64 malignant and 6 benign tumors were confirmed histopathologically ( $\vee$ Fig. 2). The malignant tumors included 28 metas- 
tases (including 25 with a colorectal primary tumor; 1 neuroendocrine tumor, 1 case of nasopharyngeal carcinoma, 1 case of breast cancer as the primary tumor), 24 HCCs, 9 CCCs, and 3 cases of gallbladder cancer. The group of benign lesions included 2 adenomas, 2 hemangiomas, $1 \mathrm{FNH}$, and 1 cyst. The size of the 70 tumors ranged from $10 \mathrm{~mm}$ to $151 \mathrm{~mm}$ with a mean of $49 \mathrm{~mm}$ $\mathrm{SD}+/-31 \mathrm{~mm}$.

For malignant tumors, the indication for surgery was always a high-grade suspicion of a malignant tumor in the liver with a curative intention $(n=64)$. 3 benign tumors were resected due to the size of the lesion ( 2 hemangiomas, 1 FNH > $100 \mathrm{~mm}$ ). 3 benign tumors were incorrectly described as suspicious for malignancy based on the preoperative imaging resulting in a decision to perform surgical resection (2 adenomas, 1 complicated cyst). MRI classified both adenomas and the complicated cyst as malignant. CEUS characterized one of the two adenomas as suspicious for malignancy.

\section{Determination of tumor status}

$63 / 70$ lesions were correctly classified as malignant or benign based on CEUS. Therefore, a sensitivity of $90 \%$ was calculated for CEUS. 7/70 diagnoses were assessed as "incorrect", including 6 cases in which the tumor status could not be clearly identified based on CEUS ( $\triangleright$ Table 1 ).

The information provided by MRI as a complementary imaging method correlated with histology in 64/70 cases resulting in a sensitivity of $91.4 \%$ for MRI. In 6/70 cases the MRI diagnosis was not identical with the histopathological diagnosis with the tumor entity not being defined in 3/70 cases ( $\bullet$ Table 2 ). No malignant lesion was incorrectly classified as benign on CEUS or MRI.

\section{Malignant lesions}

The test quality criteria for malignant lesions were as follows: For CEUS a sensitivity of $90.6 \%$, a positive predictive value (PPV) of $98.3 \%$, a specificity of $83.3 \%$, and a negative predictive value (NPV) of $83.3 \%$. MRI reached a sensitivity of $96.8 \%$, a PPV of $98.4 \%$, a specificity of $33.3 \%$, and an NPV of $33.3 \%$.

A significant difference between CEUS and MRI could not be determined $(p=1.000)$. Thus, the imaging methods were comparable with respect to the determination of tumor status ( Fig. 3, $\triangleright$ Table 3 ).

\section{Differentiation of tumors}

With respect to the differentiation of tumors, the CEUS diagnosis corresponded with the histopathological diagnosis in 49/70 cases. This resulted in a sensitivity of $70 \%$ for differential diagnosis. CEUS differential diagnosis was evaluated as "false" in 21/70 cases, while there was no differential diagnosis in 16/70 cases. MRI was correct in 55/70 cases, without a diagnosis in 9/70 cases and "false" in 15/ 70 cases. The sensitivity of MRI for the differentiation of tumors was thus $78.6 \%$. No significant difference in the diagnostic value for differential diagnosis could be determined $(p=0.210)$

\section{( $\vee$ Fig. 3, $\triangleright$ Table 3 ).}

In the evaluation of wrong diagnoses, 3 CCCs were classified incorrectly with the differentiation from metastases on CEUS and MRI being incorrect ( $\vee$ Table 4). Clear differentiation between $\mathrm{HCC}$ and CCC was not possible on MRI in 2 cases ( $\triangleright$ Table 4 ).

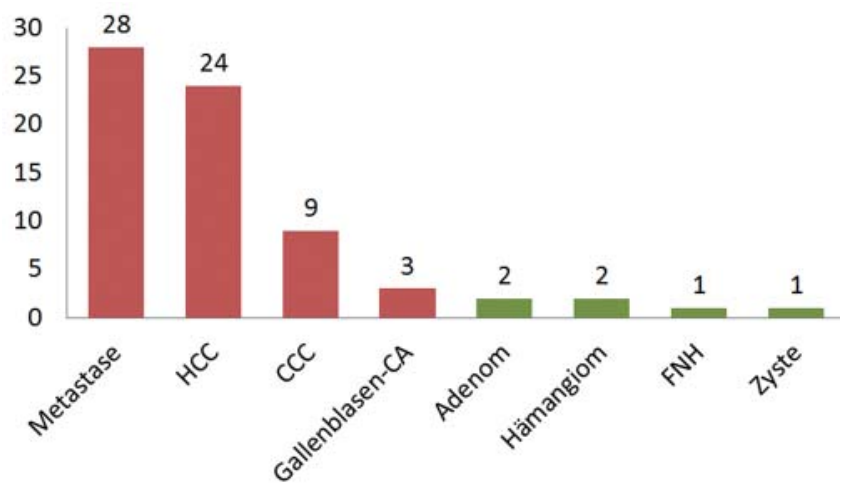

- Fig. 2 There were 64 malignant and 6 benign tumors that were histopathologically proven. For the malignant lesions there were 28 metastases (mostly colorectal cancer; 1 NET; 1 nasopharyngeal carcinoma and 1 case of breast cancer), 24 HCCs, 9 CCCs and 3 cases of gallbladder cancer. The benign lesions included 2 adenomas, 2 hemangiomas, $1 \mathrm{FNH}$ and 1 cyst.

- Table 158 malignant and 5 benign lesions were characterized correctly by CEUS. No malignant lesion was incorrectly classified as benign.

\begin{tabular}{|l|l|l|}
\hline & $\begin{array}{l}\text { malignant lesions } \\
\text { (histologically } \\
\text { confirmed) }\end{array}$ & $\begin{array}{l}\text { benign lesions } \\
\text { (histologically } \\
\text { confirmed) }\end{array}$ \\
\hline CEUS "malignant" & 58 & 1 \\
\hline CEUS "benign" & 0 & 5 \\
\hline $\begin{array}{l}\text { CEUS "definitive } \\
\text { classification not }\end{array}$ & 6 & 0 \\
\hline possible" & & \\
\hline
\end{tabular}

- Table 262 malignant and 2 benign lesions were characterized correctly by MRI. No malignant lesion was incorrectly classified as benign.

\begin{tabular}{|l|l|l|}
\hline & $\begin{array}{l}\text { malignant lesions } \\
\text { (histologically } \\
\text { confirmed) }\end{array}$ & $\begin{array}{l}\text { benign lesions } \\
\text { (histologically } \\
\text { confirmed) }\end{array}$ \\
\hline MRI "malignant" & 62 & 3 \\
\hline MRI "benign" & 0 & 2 \\
\hline $\begin{array}{l}\text { MRI "definitive } \\
\text { classification not } \\
\text { possible" }\end{array}$ & 2 & 1 \\
\hline
\end{tabular}

For the case groups HCC and metastasis, a separate comparison between CEUS and MRI was performed ( $>$ Fig. 3, $\triangleright$ Table 3):

HCC Among 24 cases of HCC, CEUS diagnosed 15/24 cases correctly (sensitivity 62.5\%). No diagnosis was made in 9/24 cases. There were no wrong diagnoses. MRI detected an HCC 


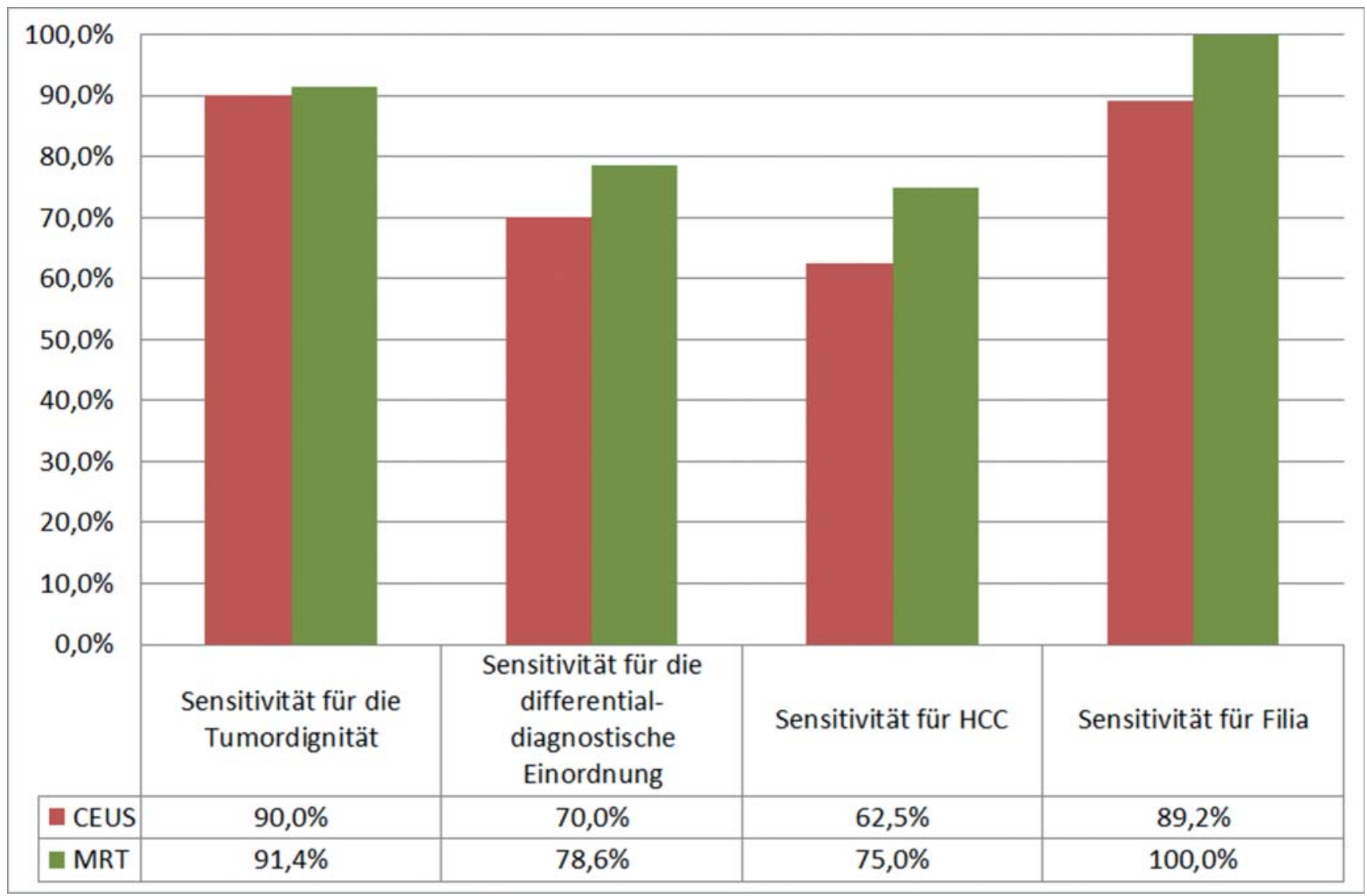

- Fig. 3 Graphic display of the sensitivity of CEUS in comparison to MRI. In terms of the determination of malignancy/benignity, CEUS had a sensitivity of $90 \%$ and MRI had a sensitivity of $91.4 \%$. For differential diagnosis, the sensitivity of CEUS was $70.0 \%$, while the sensitivity of MRI was $78.6 \%$. For the differential diagnoses "HCC" and "metastasis", a sensitivity of $62.5 \%$ and $89.2 \%$, respectively, was achieved for CEUS, while a sensitivity of $75.0 \%$ and $100 \%$, respectively, was achieved for MRI.

- Table 3 Comparison of MRI with CEUS in terms of determination of benignity/malignancy, differential diagnosis as well as determination of HCCs and metastases (McNemar test).

\begin{tabular}{|l|l|l|l|l|l|}
\hline analysis aspect & $\begin{array}{l}\text { no. of cases in } \\
\text { which an analysis } \\
\text { was possible }\end{array}$ & $\begin{array}{l}\text { number of correct } \\
\text { CEUS diagnoses }\end{array}$ & $\begin{array}{l}\text { number of correct } \\
\text { MRI diagnoses }\end{array}$ & $\begin{array}{l}\text { p-value } \\
\text { significant differ- } \\
\text { ence between CEUS } \\
\text { and MRI? }\end{array}$ \\
\hline tumor status & 70 & $63(90.0 \%)$ & $64(91.4 \%)$ & 1.000 & no \\
\hline differential diagnosis & 70 & $49(70.0 \%)$ & $55(78.6 \%)$ & 0.210 & no \\
\hline HCC lesions & 24 & $15(62.5 \%)$ & $18(89.2 \%)$ & 0.453 & no \\
\hline Metastases & 28 & $25(75.0 \%)$ & $28(100 \%)$ & 0.250 & no \\
\hline
\end{tabular}

correctly in $18 / 24$ cases (sensitivity $75.0 \%$ ), did not yield a diagnosis in 5/24 cases, and diagnosed one case incorrectly (HCC incorrectly classified as $(C C)$. There was no significant difference between CEUS and MRI regarding HCC diagnosis ( $p=0.453)$.

Metastases In the case of 28 histologically confirmed metastases, CEUS was able to correctly detect 25/28 tumors, while MRI was able to identify all 28 tumors. The sensitivity of CEUS was thus $89.2 \%$ and the sensitivity of MRI was $100 \%$. In 3/28 cases, no differential diagnosis was made based on the CEUS findings. There was no significant difference regarding the correct differential diagnosis of liver metastases $(p=0.250)$.

\section{Tumor size}

There was an average deviation regarding tumor size of $+/-3 \mathrm{~mm}$ for CEUS and +/- $4 \mathrm{~mm}$ for MRI. The macropathological measurement as the gold standard was used as the basis. The difference regarding the measurement of lesions with CEUS and MRI was 
- Table 4 Comparison of CEUS with MRI in terms of wrong diagnosis. Especially the discrimination between CCC, HCC and metastases was not correct in all cases.

\begin{tabular}{|l|l|l|}
\hline $\begin{array}{l}\text { histopathological } \\
\text { diagnosis }\end{array}$ & $\begin{array}{l}\text { incorrectly diag- } \\
\text { nosed as (CEUS) }\end{array}$ & $\begin{array}{l}\text { incorrectly diag- } \\
\text { nosed as (MRI) }\end{array}$ \\
\hline CCC $(n=3)$ & Metastasis $(n=3)$ & $\begin{array}{l}\text { Metastasis }(n=2), \\
\text { HCC }\end{array}$ \\
\hline FNH & Adenoma & - \\
\hline Gallbladder cancer & - & HCC \\
\hline Adenoma & HCC & HCC \\
\hline HCC & - & CCC \\
\hline
\end{tabular}

not significant according to the t-test for two paired samples $(p=0.579)$.

\section{Location in relation to the liver lobes}

In 68 cases the findings were able to be evaluated with respect to the location of the lesions ("only one or both liver lobes affected?"). The histopathological finding was used as the standard for determining correct assessment by CEUS and MRI. The results of preoperative CEUS and MRI corresponded to histopathology in $57 / 68$ cases (83.8\%). In 8/68 cases in which preoperative CEUS assumed lesions in only one liver lobe, MRI showed tumor in both liver lobes. In 3/68 cases in which MRI classified one liver lobe as tumor-free, preoperative CEUS disproved that finding ( $\triangleright$ Fig. 5). A significant difference between CEUS and MRI was not determined $(p=0.132)$.

Intraoperative ultrasound confirmed whether one or both lobes were affected. In 42/70 cases in which IOCEUS examination was performed in addition to CEUS and MRI, there were only 2 cases in which the CEUS findings differed from the location of the tumor determined by IOCEUS and only 1 case in which the MRI findings differed from the IOCEUS results $(4.7 \%$ and $2.3 \%$, respectively).

\section{Segmental location}

In 53 cases segment data was available both in the preoperative CEUS findings and in the MRI findings. The correct location was determined from the resection decision and the subsequent histopathological evaluation. CEUS and MRI were evaluated based on whether they were able to detect a segment containing a lesion subsequently confirmed by histology. In 14/53 cases ultrasound was able to diagnose $\geq 1$ additional segments than MRI as having "malignant infiltration" (26.4\%). MRI was able to detect more segments affected by a tumor than ultrasound in 19/53 cases $(35.8 \%$ ) ( Table 5 ). There was no significant difference between CEUS and MRI regarding segment diagnosis $(p=0.121)$.

\section{Lesion detection}

For the evaluation of lesion detection, the correct number of lesions was the maximum number visualized by an imaging modality and confirmed by histology. The number of lesions was simply specified as "multiple" in 7/70 cases on preoperative CEUS and in 8/70 cases on MRI. A concrete number of lesions were specified in the remaining cases. In 33/70 cases, both imaging methods detected the same number of lesions. In 16/70 cases, MRI identified more lesions, while ultrasound identified more lesions in $9 / 70$ cases. However, there was no significant superiority of MRI over CEUS $(p=0.083)$ ( $\vee$ Table 5$)$.

Since the examination modalities are comparable with respect to lesion detection, the value of the preoperative use of a combination of MRI and CEUS was analyzed in the following. Using the combination of imaging methods, more lesions could be detected in $9 / 70$ cases than with MRI and in $16 / 70$ cases than with CEUS. The addition of complementary preoperative imaging yielded a significant advantage both for CEUS and MRI $(p<0.001$ for CEUS and $\mathrm{p}=0.004$ for MRI).

Preoperative CEUS imaging was supplemented by IOCEUS in $42 / 70$ cases. Lesions $<10 \mathrm{~mm}$ could not be visualized intraoperatively on B-mode imaging, thus necessitating the use of CEUS.

One lesion previously identified on preoperative CEUS could not be visualized in two cases. In all other cases, all preoperatively known lesions could also be detected during intraoperative ultrasound examination.

In 37/70 cases a comparison between intraoperative ultrasound and preoperative MRI regarding lesion detection was possible. All lesions visualized on MRI were identified. Lesions that could not be diagnosed on MRI were additionally seen in 10/ 37 cases (27\%) ( $\triangleright$ Table 5 ). In the evaluation, the number and segment allocation of the tumor lesions based on IOCEUS were correlated to the histopathological finding and confirmed based on size and location.

IOCEUS proved to be advantageous on a statistically significant level $(p=0.004)$ for the detection of tumor lesions not preoperatively visualized by MRI. A significant superiority of IOCEUS regarding lesion detection was also able to be determined compared to preoperative CEUS examination $(p=0.002)$. The surgical therapy decision was modified based on the IOCEUS diagnosis in all 10/37 cases in which lesions were detected in addition to those found on preoperative MRI and the resection was expanded.

\section{Discussion}

Our current study highlights the importance of the presence of a radiologist with CEUS experience for performing intraoperative ultrasound to ensure optimized liver surgery. It was also able to be shown that MRI and preoperative contrast-enhanced ultrasound are comparable in the diagnosis of liver tumors regarding tumor entity, tumor size, and tumor location. There were no significant differences regarding detection, localization, determination of tumor status, and differential diagnosis between CEUS and MRI. Only IOCEUS provided a significant advantage with respect to the detection of lesions that could not be diagnosed by MRI and preoperative CEUS, resulting in modification of the surgical therapy decision and expansion of the resection in 10/ 37 cases $(27 \%)$.

The largest and most comprehensive analysis of CEUS and the diagnosis of liver tumors is the multicenter, prospective DEGUM study $[3-5,13]$. It was able to be shown that CEUS has high diagnostic value for all benign and malignant liver tumor entities. 

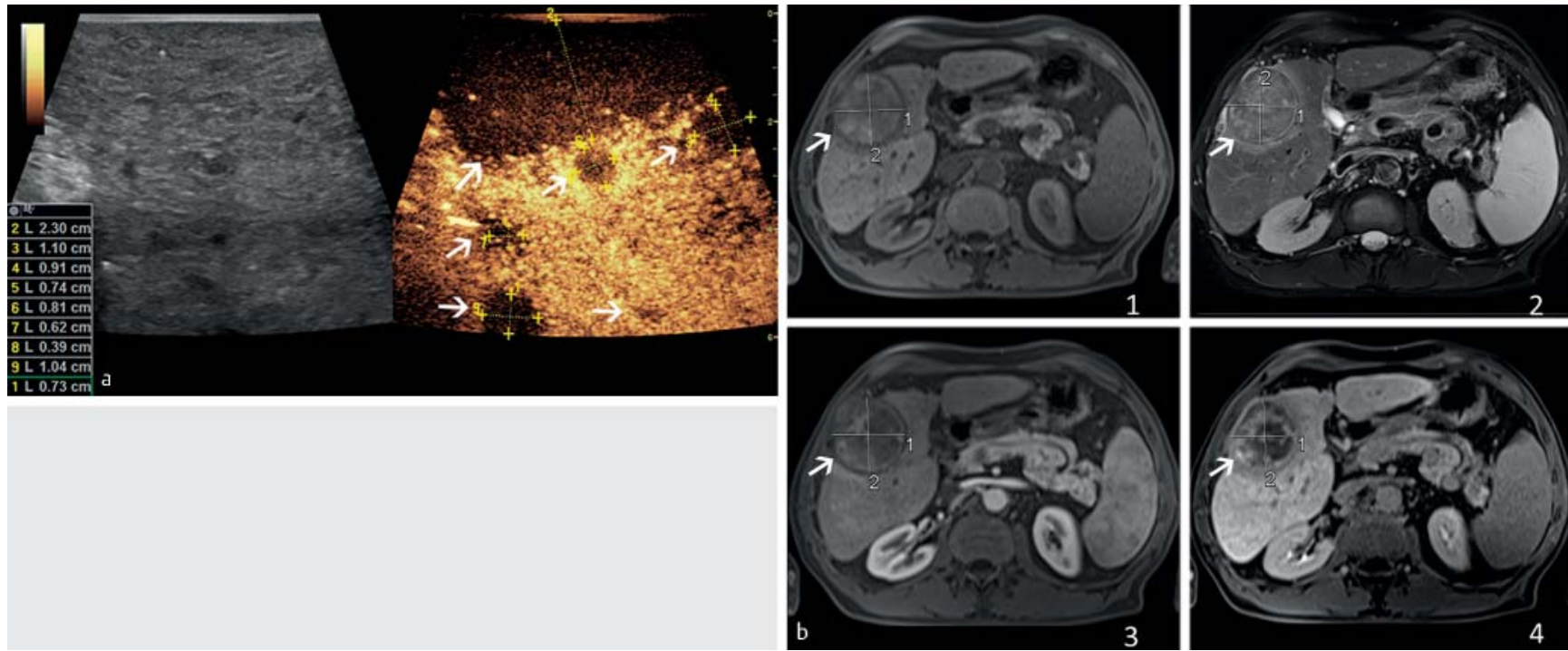

- Fig. 4 a Intraoparative display of a superficial HCC lesion $(4.8 \times 2.3 \mathrm{~cm})$ with 5 satellite lesions $(<1 \mathrm{~cm}$, arrows). In B-mode the liver tissue appears to be cirrhotic and inhomogeneous (left side). In CEUS (right) the tumor lesions and the central necrosis are clearly visualized. Multifrequency probe $(6-9 \mathrm{MHz})$, virtual convex mode, low mechanical index $(\mathrm{Ml}<0.2)$ after injection of $5 \mathrm{ml}$ SonoVue i. v., wash-out as criteria of malignancy. Arterial phase ( 8 sec. post injection). b Additional imaging using MRI. T1w (1), T2w (2), T1w vibe arterial phase (3) and T1w late phase (4) with $7 \mathrm{ml}$ Primovist. The small satellite lesions are better seen in intraoperative CEUS.
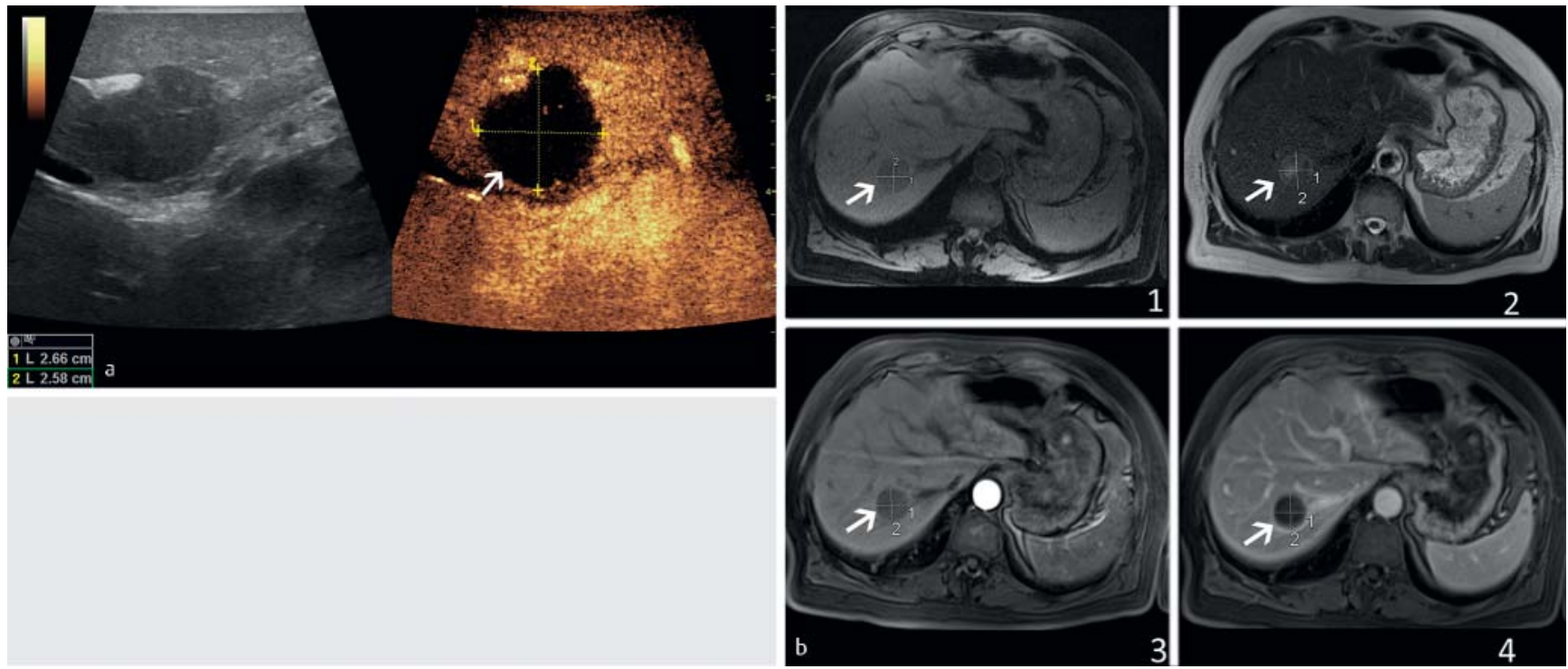

- Fig. 5 a Intraoperative display of a metastasis after colorectal cancer close to the veins $(2.5 \times 2.6 \mathrm{~cm})$ using a 6 - 9 MHz multifrequency probe, virtual convex mode, low mechanical index ( $\mathrm{Ml}<0.2)$ after injection of $5 \mathrm{ml}$ SonoVue i. v., wash-out as criteria of malignancy $(3$ minutes post injection). b Same metastasis after colorectal cancer in MRI, as seen in CEUS. T1w (1), T2w (2), T1w vibe arterial phase (3) and T1w late phase (4) with 7 ml Primovist.

Histology was obtained whenever possible. The main criterion for the detection of malignant lesions was the combination of irregular arterial vascularization and increasing wash-out to the portal venous phase. Depending on the type of tumor, diagnostic accuracy of up to $100 \%$ was able to be achieved, while accuracy of up to $>80 \%$ was achieved for most cases of HCC and CCC $[3-5,13]$.

The main criterion for benign lesions was nodular, centripetal/ centrifugal contrast enhancement usually with a lack of contrast enhancement in the late phase. Diagnostic accuracy of $100 \%$ should be achieved in the case of benign lesions. However, irregularly vascularized adenomas, atypical partially thrombosed hemangiomas, and small regenerative nodules in the case of cirrhosis were difficult to classify [10]. Differential diagnosis can even be difficult on MRI in such cases regardless of their benign genesis. Thus, in cases of doubt they require histological confirmation via biopsy or follow-up. 
- Table 5 Comparison of CEUS with MRI in terms of number of lesions and number of lobes/segments infiltrated by tumor (Wilcoxon test).

\begin{tabular}{|l|l|l|l|l|l|}
\hline analysis aspect & $\begin{array}{l}\text { no. of cases in } \\
\text { which an analysis } \\
\text { was possible }\end{array}$ & MRI > CEUS & CEUS > MRI & $\begin{array}{c}\text { p-value } \\
\text { significant differ- } \\
\text { ence between } \\
\text { CEUS and MRI? }\end{array}$ \\
\hline $\begin{array}{l}\text { liver lobe } \\
\text { number of segments }\end{array}$ & 68 & $8(11.7 \%)$ & $3(4.4 \%)$ & 0.132 & no \\
\hline $\begin{array}{l}\text { number of lesions } \\
\text { (preoperative CEUS) }\end{array}$ & 53 & $19(35.8 \%)$ & $14(26.4 \%)$ & 0.121 & no \\
\hline number of lesions (IOCEUS) & 37 & $16(27.5 \%)$ & $9(15.5 \%)$ & 0.083 & no \\
\hline
\end{tabular}
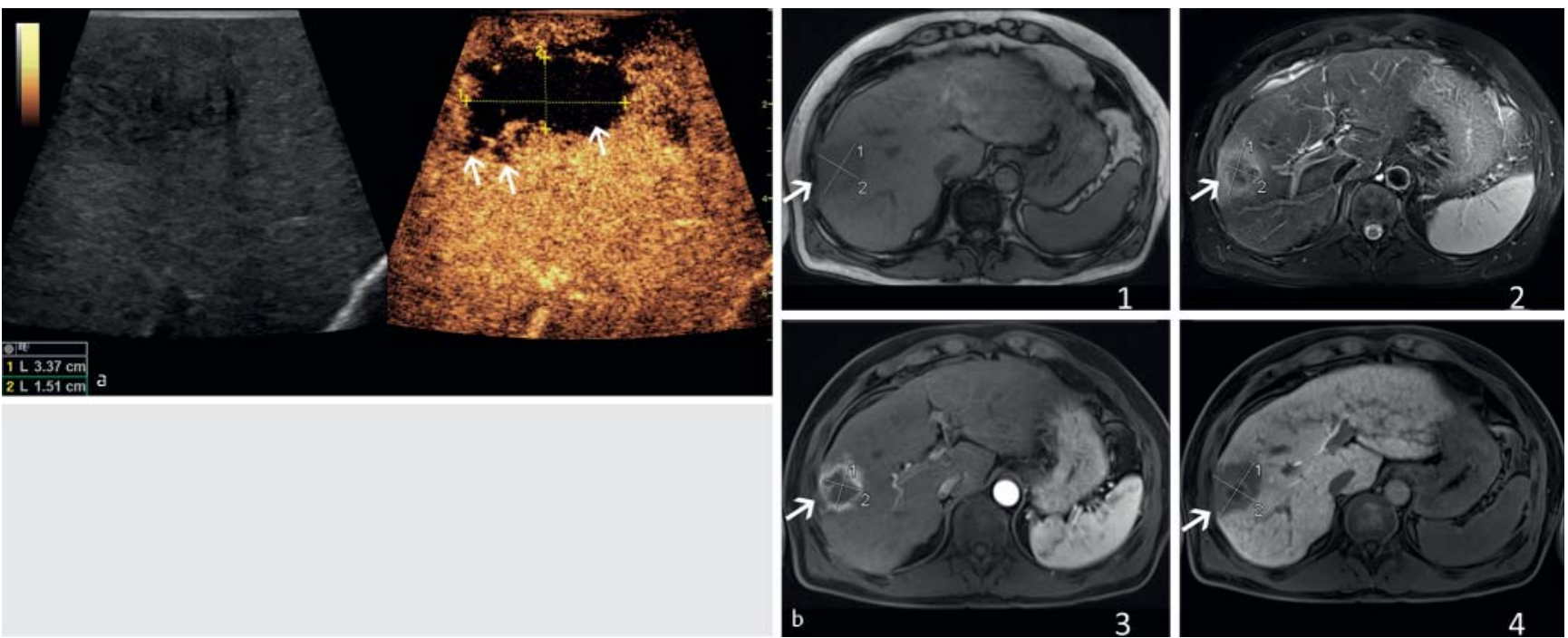

- Fig. 6 a Intraoperative display of a CCC lesion $(3.2 \times 1.6 \mathrm{~cm})$ with small satellite lesions $(<1 \mathrm{~cm}$, arrows). Performed with a $6-9 \mathrm{MHz}$ multifrequency probe, virtual convex mode and low mechanical index $(\mathrm{Ml}<0.2)$ after injection of $5 \mathrm{ml}$ SonoVue i. v., wash-out as criteria of malignancy. Arterial phase (9 seconds post injection). b Cholangiocellular carcinoma (CCC) in MRI. T1w (1), T2w (2), T1w vibe arterial phase (3) and T1w late phase (4) with $7 \mathrm{ml}$ Primovist.

Considering all tumor entities, the DEGUM study showed: CEUS is equivalent to contrast-enhanced CT of the liver and can provide advantages in the detection of small lesions $(<10 \mathrm{~mm})$ [22]. However, the comparison to MRI was not performed with liver-specific contrast agent in all cases [3-5]. This highlights the importance of this study in which the comparison to MRI involves the use of liver-specific contrast agent (Primovist $\left.{ }^{\circledR}, B A Y E R\right)$ in every case.

The results of this study have been confirmed by other current studies $[1,6-8,10,12,15,18]$. CEUS was able to achieve a sensitivity of $>80 \%$ for the differentiation between malignant and benign lesions in all specified studies. Studies that take differential diagnosis into consideration were able to identify advantages of CEUS particularly with respect to HCC diagnosis [22] as well as in cirrhotic liver tissue [8, 9]. A significant advantage of CEUS was shown for the diagnosis of colorectal liver metastases [11, 15] and for diagnostic imaging during/after chemotherapy [19, 22] and after ablation therapy $[22,23]$. In studies using CT and MRI for comparison, no significant differences regarding the determination of tumor status were able to be shown [12].

Analyses examining intraoperative contrast-enhanced ultrasound showed the possibilities of IOCEUS for detecting additional tumors and small lesions $(<10 \mathrm{~mm})[8,15,18,19]$. This was not possible in B-mode.

Intraoperative determination of the status of tumors was also important. This could only be assessed intraoperatively by CEUS, which highlights the need for intraoperative contrast administration. IOCEUS proved to have high diagnostic significance particularly in patients who underwent preoperative chemotherapy [24]. This study was able to confirm the results of other studies also in this regard in that it showed a significant advantage of IOCEUS for resection decisions compared to all preoperative imaging modalities $[15,19]$.

Limitations of the study design There are limitations of a retrospective study design, for example with respect to data collection and the analysis of causal relationships. 
Limitations of CEUS Ultrasound is limited with respect to topographic regions of the liver that are also difficult to visualize on intraoperative CEUS in some cases (subdiaphragmatic segment VIII). Moreover, special ultrasound devices and a highly experienced examiner are needed to acquire high-quality contrast-enhanced ultrasound scans. In addition, ultrasound is a depth-dependent imaging modality that can reach its limits particularly in overweight patients. IOCEUS can ensure good visualization of the liver even in overweight patients but is subject to time constraints due to the surgical situation.

Limitations of MRI MRI is dependent on the imaging technology and the field strength. Breathing artifacts can affect image quality particularly in liver diagnosis as a result of the proximity to the diaphragm. Because it is a time-intensive examination, good patient compliance is necessary. Moreover, absolute contraindications must be taken into consideration (i. e., older generation pacemakers, magnetizable objects) and contraindications in relation to gadolinium-containing intravenous contrast agent (from nephropathies to the rarely occurring nephrogenic systemic fibrosis) [24].

Finally a particularly valuable aspect of this study is that a comparative analysis of the imaging modalities CEUS and MRI in relation to liver tumors was only performed in cases in which the gold standard of a histopathological tumor diagnosis was available. Since this constellation only applied in 70 of 317 cases, this explains the low number of included cases but ensures the high quality and significance of this analysis. Moreover, not only preoperative imaging but also intraoperative imaging was included in this study, thus representing a further advantage. Based on the results of this study, a combination of CEUS and MRI with liver-specific contrast agent is recommended in the case of suspicion of a malignant tumor in the liver. Intraoperative CEUS can be an important part of resection planning.

Further prospective studies are needed to prove the value of intraoperative CEUS imaging in liver surgery.

\section{Conclusion}

In the preoperative diagnosis of liver tumors, CEUS is a dynamic imaging method with high diagnostic significance regarding dynamic tumor vascularization for evaluating the entity, size, and location of liver tumors. A combination of MRI and CEUS is highly advantageous for the detection of lesions. Intraoperative ultrasound with CEUS provides a significant advantage for the detection of lesions not diagnosed preoperatively and thus makes an important contribution to surgical therapy decisions.

\section{CLINICAL RELEVANCE OF THE STUDY}

- CEUS and MRI with liver-specific contrast agent (Primovist $^{\circledR}$ ) are equivalent preoperative imaging modalities with respect to the detection and status evaluation of liver tumors.

- Preoperative complementary imaging with MRI supplemented by CEUS is highly advantageous for detecting liver lesions compared to the use of only one of the two methods.

- IOCEUS can detect lesions and their dimensions not visualized by preoperative imaging when performed by an experienced examiner so that resection can be expanded as needed.

\section{Conflict of Interest}

No conflict of interest has been declared by the author(s).

\section{References}

[1] Reis MACR, Baroni RH. Liver-specific magnetic resonance contrast medium in the evaluation of chronic liver disease. Einstein (Sao Paulo, Brazil) 2015; 13: 326-329

[2] Hammerstingl R, Huppertz A, Breuer J et al. Diagnostic efficacy of gadoxetic acid (Primovist)-enhanced MRI and spiral CT for a therapeutic strategy: comparison with intraoperative and histopathologic findings in focal liver lesions. European radiology 2008; 18: 457-467

[3] Seitz K, Bernatik T, Strobel D et al. Contrast-enhanced ultrasound (CEUS) for the characterization of focal liver lesions in clinical practice (DEGUM Multicenter Trial): CEUS vs. MRI - a prospective comparison in 269 patients. Ultraschall in der Medizin (Stuttgart, Germany: 1980) 2010; 31 : $492-499$

[4] Seitz K, Strobel D, Bernatik T et al. Contrast-Enhanced Ultrasound (CEUS) for the characterization of focal liver lesions - prospective comparison in clinical practice: CEUS vs. CT (DEGUM multicenter trial). Parts of this manuscript were presented at the Ultrasound Dreilandertreffen 2008, Davos. Ultraschall in der Medizin (Stuttgart, Germany: 1980) 2009; 30: $383-389$

[5] Strobel D, Seitz K, Blank W et al. Contrast-enhanced ultrasound for the characterization of focal liver lesions - diagnostic accuracy in clinical practice (DEGUM multicenter trial). Ultraschall in der Medizin (Stuttgart, Germany: 1980) 2008; 29: 499-505

[6] Carvalho CF, Chammas MC, Souza OC et al. Elastography and Contrastenhanced Ultrasonography in the Early Detection of Hepatocellular Carcinoma in an Experimental Model of Nonalcoholic Steatohepatitis. Journal of clinical and experimental hepatology 2013; 3: 96-101

[7] Chiorean L, Cantisani V, Jenssen C et al. Focal masses in a non-cirrhotic liver: The additional benefit of CEUS over baseline imaging. European journal of radiology 2015; 84: 1636-1643

[8] Jung EM, Platz Batista da Silva N, Jung W et al. Is Strain Elastography (IO-SE) Sufficient for Characterization of Liver Lesions before Surgical Resection - Or Is Contrast Enhanced Ultrasound (CEUS) Necessary? PloS one 2015; 10: e0123737

[9] Jung EM, Wiggermann P, Stroszczynski C et al. Ultrasound diagnostics of diffuse liver diseases. Der Radiologe 2012; 52: 706 - 716

[10] Wu W, Chen M, Yan K et al. Evaluation of contrast-enhanced ultrasound for diagnosis of dysplastic nodules with a focus of hepatocellular carcinoma in liver cirrhosis patients. Chinese journal of cancer research = Chung-kuo yen cheng yen chiu 2015; 27: 83-89 
[11] Albrecht T, Hohmann J, Oldenburg A et al. Detection and characterisation of liver metastases. European radiology 2004; 14: P25 - P33

[12] Sporea I, Badea R, Popescu A et al. Contrast-enhanced ultrasound (CEUS) for the evaluation of focal liver lesions - a prospective multicenter study of its usefulness in clinical practice. Ultraschall in der Medizin (Stuttgart, Germany: 1980) 2014; 35: 259-266

[13] Strobel D, Bernatik T, Blank W et al. Diagnostic accuracy of CEUS in the differential diagnosis of small $(<1=20 \mathrm{~mm})$ and subcentimetric $(<1=$ $10 \mathrm{~mm}$ ) focal liver lesions in comparison with histology. Results of the DEGUM multicenter trial. Ultraschall in der Medizin (Stuttgart, Germany: 1980) 2011; 32: 593-597

[14] Jung EM, Clevert DA, Schreyer AG et al. Evaluation of quantitative contrast harmonic imaging to assess malignancy of liver tumors: a prospective controlled two-center study. World journal of gastroenterology 2007; 13: 6356-6364

[15] Donadon M, Costa G, Torzilli G. State of the art of intraoperative ultrasound in liver surgery: current use for staging and resection guidance. Ultraschall in der Medizin (Stuttgart, Germany: 1980) 2014; 35: 500 511 quiz 512-513

[16] Torzilli G, Botea F, Donadon M et al. Criteria for the selective use of contrast-enhanced intra-operative ultrasound during surgery for colorectal liver metastases. HPB: the official journal of the International Hepato Pancreato Biliary Association 2014; 16: $994-1001$

[17] Leen E, Ceccotti P, Moug S] et al. Potential value of contrast-enhanced intraoperative ultrasonography during partial hepatectomy for metastases: an essential investigation before resection? Annals of surgery 2006; $243: 236-240$
[18] Jung EM, Ross C], Rennert ] et al. Characterization of microvascularization of liver tumor lesions with high resolution linear ultrasound and contrast enhanced ultrasound (CEUS) during surgery: First results. Clinical hemorheology and microcirculation 2010; 46: 89-99

[19] Arita J, Ono Y, Takahashi M et al. Usefulness of contrast-enhanced intraoperative ultrasound in identifying disappearing liver metastases from colorectal carcinoma after chemotherapy. Annals of surgical oncology 2014; 21: \$390-\$397

[20] Hyvelin ], Tardy I, Arbogast C et al. Use of ultrasound contrast agent microbubbles in preclinical research: recommendations for small animal imaging. Investigative radiology 2013; 48: 570-583

[21] Wildner D, Pfeifer L, Goertz RS et al. Dynamic contrast-enhanced ultrasound (DCE-US) for the characterization of hepatocellular carcinoma and cholangiocellular carcinoma. Ultraschall in der Medizin (Stuttgart, Germany: 1980) 2014; 35: $522-527$

[22] Palmieri VO, Santovito D, Marano G et al. Contrast-enhanced ultrasound in the diagnosis of hepatocellular carcinoma. La Radiologia medica 2015; 120: $627-633$

[23] Granata V, de Lutio di Castelguidone E, Fusco R et al. Irreversible electroporation of hepatocellular carcinoma: preliminary report on the diagnostic accuracy of magnetic resonance, computer tomography, and contrast-enhanced ultrasound in evaluation of the ablated area. La Radiologia medica 2016; 121: 122 - 131

[24] Soyer P, Dohan A, Patkar D et al. Observational study on the safety profile of gadoterate meglumine in 35499 patients: The SECURE study. Journal of magnetic resonance imaging: JMRI 2016 